\title{
A família Orchidaceae na Serra do Japi, São Paulo, Brasil
}

\author{
Emerson Ricardo Pansarin ${ }^{1} \&$ Ludmila Mickeliunas Pansarin ${ }^{2}$
}

\begin{abstract}
RESUMO
(A família Orchidaceae na Serra do Japi, São Paulo, Brasil) Este estudo apresenta o inventário das espécies de orquídeas ocorrentes na Serra do Japi, no estado de São Paulo. A região é composta por áreas de floresta mesófila estacional semidecídua baixo montana e de altitude e afloramentos rochosos. A família está representada por 125 espécies, distribuídas em 61 gêneros. $O$ gênero mais representativo é Epidendrum (10 spp.), seguido de Oncidium e Habenaria (9 spp. cada). A maioria das espécies (79 spp., 63,2\%) é epífita, sendo que 40 espécies (32\%) são terrícolas, 31 espécies. (24,8\%) são rupícolas, duas são hemiepífitas e apenas uma é saprofítica. A floresta mesófila estacional semidecídua é o tipo de vegetação que abriga o maior número de espécies ( 88 spp., 70,9\%). A maioria das espécies floresce no verão, entre dezembro e março. Duas espécies, Habenaria sp. e Acianthera sp. provavelmente são novas para a ciência. A Serra do Japi, por estar em uma zona de transição entre a as florestas ombrófilas (Serra do Mar) e as florestas estacionais semideciduais do planalto paulista, abriga espécies de ambas as formações. Apesar da grande diversidade de Orchidaceae na Serra do Japi, a região sofre com a intervenção humana. A preservação e o estudo integrado da Serra do Japi é uma urgente necessidade científica, com reflexos sociais, econômicos e preservacionistas.
\end{abstract}

Palavras-chave: conservação, ecótono, floresta mesófila estacional semidecídua, levantamento florístico.

\section{AbStract}

(The family Orchidaceae in the Serra do Japi, São Paulo, Brazil) This study reports the floristic survey of orchid species occurring in the Serra do Japi, State of São Paulo, Southeastern Brazil. The region is characterized mainly by semi-deciduous mesophytic lowland and altitude forests and rocky outcrops. The family is characterized by 125 species distributed among 61 genera. The most representative genus is Epidendrum (10 spp.), followed by Oncidium and Habenaria (both with 9 spp.). Most of the species (79 spp., 63.2\%) occurs as epiphytes, while 40 species (32\%) are terrestrial, 31 species (24.8\%) are rupicolous, two are hemi-epiphytes and only one is a saprophyte. The semi-deciduous mesophytic forest has the highest occurrence of species of Orchidaceae, with 70.9\% (88 spp.) of the species. The majority of species flower in summer, between December and March. Two species, Habenaria sp. and Acianthera sp. were not identified and are possibly new to science. The Serra do Japi is strategically placed in the transition between interior semi-deciduous mesophytic forests and the Atlantic forest, presenting species from both formations. Although the orchid diversity is high, the region is affected by anthropogenic disturbance. The preservation and the integrated study is an urgent necessity, with social, economic and preservationist reflexes.

Key words: conservation, ecotone, floristic survey, semi-deciduous mesophytic forests.

\section{INTRODUÇÃo}

Orchidaceae abrange cerca de $7 \%$ das angiospermas, sendo considerada uma das maiores famílias desse grupo (Dressler 1993). Atwood (1986) estimou que a família possui cerca de 20.000 espécies distribuídas por todo o mundo, apresentando maior riqueza nas regiões tropicais. De acordo com Pabst \& Dungs (1975), o Brasil possui cerca de 2.300 espécies distribuídas em 191 gêneros. Atualmente acredita-se que esse número seja aproximadamente de 2.400 espécies para o território brasileiro (Barros 1996). Espécies de Orchidaceae podem ser encontradas em todas as formações vegetacionais brasileiras (Hoehne 1949). A grande capacidade adaptativa das orquídeas pode ser explicada, em parte, pelas várias formas das estruturas vegetativas presentes na família, as quais podem representar diferentes estratégias relacionadas com a obtenção e reserva de água e nutrientes. Caules intumescidos formando pseudobulbos,

Artigo recebido em 06/2007. Aceito para publicação em 10/2007.

${ }^{1}$ Universidade de São Paulo, Faculdade de Filosofia, Ciências e Letras de Ribeirão Preto, Departamento de Biologia, 14040-901, Ribeirão Preto, SP. epansarin@uol.com.br

${ }^{2}$ Universidade Estadual de Campinas, Programa de Pós-graduação em Biologia Vegetal, Departamento de Botânica, Instituto de Biologia, C.P. 6109, 13083-970, Campinas, SP. colax@ pop.com.br 
folhas carnosas, raízes dotadas de velame e o próprio crescimento em touceiras, permitindo o acúmulo de matéria orgânica, são algumas dessas estratégias (Hoehne 1949).

A Serra do Japi é uma das últimas regiões de mata contínua do interior do estado de São Paulo, com algumas áreas de vegetação primária bem preservadas em função, principalmente, da acidentada topografia (Morellato 1992). Sua posição geográfica peculiar, entre as florestas ombrófilas a leste (Serra do Mar) e as florestas mesófilas estacionais semidecíduas do planalto paulista a oeste, caracteriza uma região ecotonal, permitindo a ocorrência de um número elevado de espécies de ambas as formações (Leitão Filho 1992). Um estudo florístico realizado em um fragmento da Serra do Japi evidenciou elevado número de espécies arbóreas (303) (Leitão Filho 1992). Até o presente estudo nenhum inventário envolvendo espécies herbáceas havia sido realizado na região.

Em virtude de sua localização, entre dois grandes centros (Campinas e São Paulo) e circundada por regiões densamente povoadas, a Serra do Japi vem sofrendo, ao longo de praticamente toda sua extensão, grande interferência por ação antrópica. Em função desse mesmo fator, a flora e, como conseqüência a fauna da região, sofrem alterações. Além das alterações antrópicas, que provocam diferenças na vegetação, existem aquelas naturais que são devidas às diferenças de solo, de umidade e de altitude que contribuem para caracterizar floristicamente algumas áreas da Serra. Devido à heterogeneidade da vegetação, à presença de um grande número de riachos e à topografia da região, tem-se como conseqüência uma grande variação de microclimas. A diversidade de microclimas e da vegetação que, em muitos casos, é utilizada como refúgio ou fonte de alimentação, são propícias para a presença de um grande número de animais. A soma desses fatores faz da Serra do Japi uma região muito importante em termos de preservação e banco genético (Morellato 1992).
Embora as regiões de florestas mesófilas estacionais semidecíduas estejam sendo devastadas no interior do estado de São Paulo, muitas vezes pelo avanço de lavouras de canade-açúcar, muito pouco tem sido feito com relação ao conhecimento da diversidade florística e/ou conservação dessas áreas. Além disso, quando se refere à família Orchidaceae, poucos estudos de florística são realizados abrangendo esse tipo de formação no estado (para uma exceção veja Cardoso \& Israel 2005). Normalmente esses estudos são realizados em áreas de floresta ombrófila (e.g. Barros 1983; 1991; Ribeiro 1992), embora em Minas Gerais levantamentos florísticos para a família em regiões de florestas semidecíduas sejam mais freqüentes (e.g. Oliveira Filho \& Machado 1993; Oliveira Filho et al. 1994; Menini Neto et al. 2004a; 2004b). Com base nesses fatos, o presente estudo tem como principal objetivo inventariar as espécies de orquídeas presentes na Serra do Japi, além de acompanhar a fenologia de floração e verificar o habitat e a ocorrência de cada espécie em ambiente natural.

\section{Material e métodos \\ Caracterização da área}

O estudo foi realizado na Serra do Japi, localizada no estado de São Paulo, entre as coordenadas $37^{\circ} 25^{\prime} 818^{\prime \prime} \mathrm{N}, 122^{\circ} 05^{\prime} 36^{\prime \prime} \mathrm{O}$ (Fig. 1). A região apresenta aproximadamente $354 \mathrm{~km}^{2}$ e abrange quatro municípios: Cabreúva, Cajamar, Jundiaí e Pirapora do Bom Jesus (Morellato 1992). A Serra do Japi é caracterizada por altitudes que variam entre 700 e $1.300 \mathrm{~m}$, o que condiciona temperaturas médias anuais entre $15,7^{\circ} \mathrm{C}$ e $19,2^{\circ} \mathrm{C}$, nas partes mais altas e mais baixas, respectivamente. A média de precipitação anual é de aproximadamente $1.600 \mathrm{~mm}$, sendo o período de chuvas mais concentrado entre a primavera e o verão (Pinto 1992). A região da Serra do Japi apresenta áreas de floresta mesófila estacional semidecídua (700-900 m), floresta mesófila estacional semidecídua de altitude (900-1.300 m) e esparsos enclaves de lajeados rochosos 
(Leitão Filho 1992). Ao longo das florestas mesófilas estacionais semidecíduas ocorre um variado número de espécies que podem ser encontradas em regiões de floresta ombrófila, isso sucedendo, particularmente, com espécies que demonstram preferência pelas regiões mais altas da Serra do Japi. Os lajeados rochosos têm composição florística própria, sem influência das florestas da região, tendo sido interpretados como relictos de climas mais secos (Leitão Filho 1992).

\section{Trabalho de campo e laboratório}

O inventário das Orchidaceae da Serra do Japi foi realizado através de coletas aleatórias e que abrangeram toda sua extensão. A área foi percorrida desde janeiro de 1998 até junho de 2005 para coleta do material botânico e obtenção de informações sobre período de floração, habitat e formas de vida das espécies. A ocorrência de cada espécie foi estimada de forma visual. As visitas ao campo foram geralmente mensais, sendo intensificadas (semanais) em várias etapas do trabalho. Os espécimes em floração coletados durante as excursões de campo foram prensados, secos em estufa e estão incorporados ao acervo do herbário da Universidade Estadual de Campinas (UEC). O material testemunho está listado na Tabela 1.

\section{Identificação das espécies}

Para a identificação dos táxons foram utilizadas as principais obras de referência na taxonomia das Orchidaceae brasileiras (Cogniaux 1893-1896; 1898-1902; 1904-1906; Hoehne 1940; 1942; 1945; 1953; Pabst \& Dungs 1975; 1977; Sprunger et al. 1996) e alguns trabalhos mais específicos para a família (e.g. Pabst 1950; Garay 1977; 1980; Hágsater 1993; Christenson 1988; 1996; van den Berg \& Chase 2001). A divisão em subfamílias foi apresentada de acordo com Chase et al. (2003). A determinação das autoridades taxonômicas foi realizada de acordo com Kew Monocot World Checklist (www.kew.org/wcsp). Os padrões de distribuição das espécies foram determinados de acordo com Kew Monocot World Checklist (www.kew.org/wcsp) e W3 Tropicos (www.mobot.mobot.og/W3T).

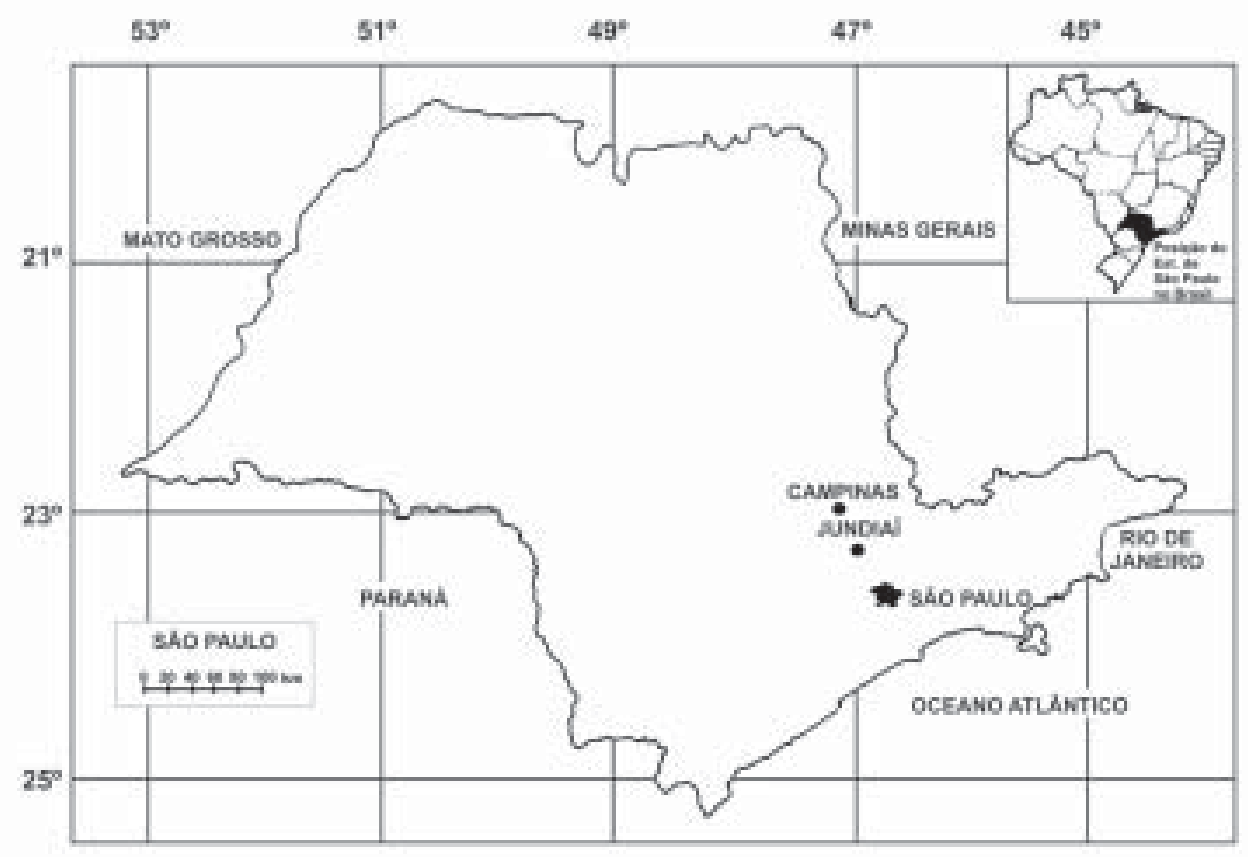

Figura 1 - Localização de Jundiaí, município que abrange a maior parte da Serra do Japi, no estado de São Paulo. Baseado em Morellato (1992). 
Tabela 1 - Espécies de Orchidaceae ocorrentes na Serra do Japi. Forma de vida: E = epífita, HE = hemiepífita, $\mathrm{R}=$ rupícola, $\mathrm{T}=$ terrícola, $\mathrm{S}=$ saprofítica. Habitat: FMES = floresta mesófila estacional semidecídua, FMESA = floresta mesófila estacional semidecídua de altitude, MG = mata de galeria, $\mathrm{LR}=$ lajeado rochoso, $\mathrm{LP}=$ local perturbado. Ocorrência: $\mathrm{C}=$ comum, $\mathrm{PC}=$ pouco comum, $\mathrm{RR}=$ rara, $\mathrm{MR}=$ muito rara. Letras entre parênteses $=$ Tipo de forma de vida menos freqüente.

\begin{tabular}{|c|c|c|c|c|c|}
\hline Espécies & $\begin{array}{l}\text { Forma } \\
\text { de vida }\end{array}$ & Habitat & Floração & Ocorrência & $\begin{array}{l}\text { Material } \\
\text { testemunho }\end{array}$ \\
\hline $\begin{array}{l}\text { Acianthera aphthosa (Lindl.) } \\
\text { Pridgeon \& M.W.Chase }\end{array}$ & $\mathrm{E}$ & $\begin{array}{l}\text { FMES/ } \\
\text { FMESA }\end{array}$ & Set-Out & $\mathrm{C}$ & $97 / 75^{\mathrm{a}}, 1093^{\mathrm{a}}, 1098^{\mathrm{a}}$ \\
\hline $\begin{array}{l}\text { Acianthera auriculata (Lindl.) } \\
\text { Pridgeon \& M.W. Chase }\end{array}$ & E & FMES & Nov-Fev & $\mathrm{PC}$ & $97 / 90^{\mathrm{a}}, 834^{\mathrm{a}}, 1106^{\mathrm{a}}$ \\
\hline $\begin{array}{l}\text { Acianthera leptotifolia (Barb. } \\
\text { Rodr.) Pridgeon \& M.W.Chase }\end{array}$ & $\mathrm{E}$ & FMES & Nov-Jun & $\mathrm{PC}$ & $563^{\mathrm{a}}, 570^{\mathrm{a}}$ \\
\hline $\begin{array}{l}\text { Acianthera luteola (Lindl.) } \\
\text { Pridgeon \& M.W.Chase }\end{array}$ & $\mathrm{E}$ & FMES & Mar-Abr & $\mathrm{RR}$ & $846^{\mathrm{a}}, 1144^{\mathrm{a}}, 1147^{\mathrm{a}}$ \\
\hline $\begin{array}{l}\text { Acianthera saundersiana } \\
\text { (Rchb.f.) Pridgeon \& M.W.Chase }\end{array}$ & $\mathrm{E}$ & FMES & Nov-Fev & $\mathrm{PC}$ & $\begin{array}{l}411^{\mathrm{a}}, 717^{\mathrm{a}}, 998^{\mathrm{a}}, 1060^{\mathrm{a}}, \\
1138^{\mathrm{a}}\end{array}$ \\
\hline $\begin{array}{l}\text { Acianthera saurocephala (Lodd.) } \\
\text { Pridgeon \& M.W.Chase }\end{array}$ & $\mathrm{E}$ & $\begin{array}{l}\text { FMES/ } \\
\text { FMESA }\end{array}$ & Out-Dez & $\mathrm{RR}$ & $728^{\mathrm{a}}, 1100^{\mathrm{a}}$ \\
\hline Acianthera sp. & $\mathrm{E}$ & FMES & Out-Nov & $\mathrm{PC}$ & $551^{\mathrm{a}}, 1097^{\mathrm{a}}, 1163^{\mathrm{a}}$ \\
\hline $\begin{array}{l}\text { Aspidogyne hylibates (Rchb.f.) } \\
\text { Garay }\end{array}$ & $\mathrm{T}$ & FMESA & Fev-Mar & MR & $837^{\mathrm{a}}$ \\
\hline $\begin{array}{l}\text { Aspidogyne metallescens } \\
\text { (Barb.Rodr.) Garay }\end{array}$ & $\mathrm{T}$ & FMES & Ago-Set & $\mathrm{PC}$ & $1073^{\mathrm{a}}$ \\
\hline $\begin{array}{l}\text { Baptistonia fimbriata (Lindl.) } \\
\text { Chiron \& V.P. Castro }\end{array}$ & $\mathrm{E}$ & FMES & Nov-Dez & $\mathrm{MC}$ & $968^{\mathrm{a}}, 927^{\mathrm{a}}$ \\
\hline $\begin{array}{l}\text { Baptistonia pubes (Lindl.) } \\
\text { Chiron \& V.P. Castro }\end{array}$ & $\mathrm{E}$ & $\begin{array}{l}\text { FMES/ } \\
\text { FMESA }\end{array}$ & Ago-Set & $\mathrm{MC}$ & $547^{\mathrm{a}}, 1095^{\mathrm{a}}, 1078^{\mathrm{a}}$ \\
\hline $\begin{array}{l}\text { Baptistonia sarcodes (Lindl.) } \\
\text { Chiron \& V.P. Castro }\end{array}$ & $\mathrm{E}$ & FMES & Nov-Dez & $\mathrm{PC}$ & $97 / 77^{\mathrm{a}}$ \\
\hline $\begin{array}{l}\text { Barbosella cogniauxiana (Speg. } \\
\text { \& Kraenzl.) Schltr. }\end{array}$ & $\mathrm{R}$ & FMES & Jan-Mar & MR & $708^{\mathrm{a}}$ \\
\hline $\begin{array}{l}\text { Bifrenaria harrisoniae (Hook.) } \\
\text { Rchb.f. }\end{array}$ & $\mathrm{R}$ & $\mathrm{LR}$ & Out-Dez & MR & $1239^{\mathrm{a}}$ \\
\hline $\begin{array}{l}\text { Brasiliorchis chrysantha } \\
\text { R.Singer, S.Koehler \& Carnevali }\end{array}$ & $\mathrm{R}$ & LR/FMES & Setembro & $\mathrm{C}$ & $1086^{\mathrm{a}}$ \\
\hline $\begin{array}{l}\text { Brasiliorchis consanguinea } \\
\text { (Klotzsch) R.Singer, S.Koehler } \\
\text { \& Carnevali }\end{array}$ & $\mathrm{R} / \mathrm{E}$ & FMES/LR & Dezembro & $\mathrm{PC}$ & $969^{\mathrm{a}}, 1096^{\mathrm{a}}, 930^{\mathrm{a}}, 1110^{\mathrm{a}}$ \\
\hline $\begin{array}{l}\text { Brasiliorchis gracilis (Lodd.) } \\
\text { R.Singer, S.Koehler \& Carnevali }\end{array}$ & $\mathrm{E}$ & FMES & Set-Out & $\mathrm{RR}$ & $729^{a}$ \\
\hline $\begin{array}{l}\text { Brasiliorchis picta (Hook.) } \\
\text { R.Singer, S.Koehler \&Carnevali }\end{array}$ & $\mathrm{R} / \mathrm{E}$ & LR/FMES & Jul-Set & $\mathrm{MC}$ & $211^{\mathrm{a}}, 1153^{\mathrm{a}}$ \\
\hline $\begin{array}{l}\text { Bulbophyllum glutinosum (Barb. } \\
\text { Rodr.) Cogn. }\end{array}$ & $\mathrm{E}$ & FMESA & Abr-Mai & $\mathrm{PC}$ & $1151^{\mathrm{a}}$ \\
\hline
\end{tabular}




\begin{tabular}{|c|c|c|c|c|c|}
\hline Espécies & $\begin{array}{l}\text { rma } \\
\text { vida }\end{array}$ & Habitat & Floração & Ocorrência & $\begin{array}{l}\text { Material } \\
\text { testemunho }\end{array}$ \\
\hline Bulbophyllum ipanemense Hoehne & $\mathrm{E} /(\mathrm{R})$ & LR/FMES & Fev-Abr & $\mathrm{C}$ & $851^{\mathrm{a}}$ \\
\hline Bulbophyllum punctatum Barb. Rodr. & $\mathrm{E}$ & FMES & Outubro & $\mathrm{RR}$ & $730^{\mathrm{a}}$ \\
\hline Bulbophyllum regnellii Rchb.f. & $\mathrm{E}$ & FMES & Fev-Mar & MR & $402^{\mathrm{a}}, 997^{\mathrm{a}}$ \\
\hline $\begin{array}{l}\text { Campylocentrum micranthum } \\
\text { (Lindl.) Rolfe }\end{array}$ & $\mathrm{E}$ & FMES & Mar-Abr & $\mathrm{RR}$ & $1238^{\mathrm{a}}$ \\
\hline Capanemia superflua (Rchb.f.) Garay & $\mathrm{E}$ & FMESA & Out-Nov & MR & $724^{\mathrm{a}}$ \\
\hline Capanemia thereziae Barb. Rodr. & $\mathrm{E}$ & FMESA & Mar-Abr & $\mathrm{RR}$ & $424^{\mathrm{a}}$ \\
\hline Catasetum cernuит (Lindl.) Rchb.f. & $\mathrm{E} /(\mathrm{R})$ & FMES & Out-Nov & $\mathrm{MC}$ & $548^{\mathrm{a}}$ \\
\hline Cattleya loddigesii Lindl. & $\mathrm{E} /(\mathrm{R})$ & $\begin{array}{l}\text { FMES/ } \\
\text { FMESA/MC }\end{array}$ & $\begin{array}{l}\text { Dez-Mar } \\
\text { IG }\end{array}$ & $\mathrm{PC}$ & $553^{\mathrm{a}}, 928^{\mathrm{a}}$ \\
\hline $\begin{array}{l}\text { Christensonella cogniauxiana } \\
\text { (Hoehne) Szlach.,Mytnik, Górniak } \\
\text { \& Emiszek. }\end{array}$ & $\mathrm{E}$ & FMES & Out-Nov & $\mathrm{C}$ & $1237^{\mathrm{a}}$ \\
\hline $\begin{array}{l}\text { Christensonella ferdinandiana } \\
\text { (Barb. Rodr.) Szlach., Mytnik, } \\
\text { Górniak \& Emiszek. }\end{array}$ & $\mathrm{E}$ & FMES & Ago-Set & $\mathrm{PC}$ & $1068^{\mathrm{a}}$ \\
\hline $\begin{array}{l}\text { Christensonella pachyphylla } \\
\text { (Schltr. ex Hoehne)Szlach., Mytnik, } \\
\text { Górniak \& (Emiszek. }\end{array}$ & $\mathrm{R} / \mathrm{E}$ & FMES & Ago-Out & MC & $\begin{array}{l}97 / 76^{\mathrm{a}}, 839^{\mathrm{a}}, 1101^{\mathrm{a}}, \\
1104^{\mathrm{a}}, 1069^{\mathrm{a}}, 1081^{\mathrm{a}}, \\
1107^{\mathrm{a}}\end{array}$ \\
\hline $\begin{array}{l}\text { Christensonella pumila (Hook.) } \\
\text { Szlach., Mytnik,Górniak \& Emiszek. }\end{array}$ & $\mathrm{E}$ & FMES & Ago-Set & $\mathrm{C}$ & $554^{\mathrm{a}}$ \\
\hline Cirrhaea dependens (Lodd.) Loudon & $\mathrm{R}$ & FMES & Dez & MR & $97 / 95^{\mathrm{a}}, 926^{\mathrm{a}}$ \\
\hline Corymborkis flava (Sw.) Kuntze & $\mathrm{T}$ & FMES & Ago-Fev & $\mathrm{PC}$ & $409^{\mathrm{a}}$ \\
\hline Cyclopogon atroviridis Barb. Rodr. & $\mathrm{T}$ & FMES & Set & PC & $1075^{\mathrm{a}}$ \\
\hline $\begin{array}{l}\text { Cyclopogon calophyllus (Barb. } \\
\text { Rodr.) Barb. Rodr. }\end{array}$ & $\mathrm{T} /(\mathrm{R})$ & FMESA & Ago-Set & PC & $556^{\mathrm{a}}, 1074^{\mathrm{a}}$ \\
\hline $\begin{array}{l}\text { Cyclopogon chloroleucus } \\
\text { (Barb. Rodr.) Schltr. }\end{array}$ & $\mathrm{T}$ & FMESA & Set-Out & $\mathrm{C}$ & $552^{\mathrm{a}}, 902^{\mathrm{a}}, 1083^{\mathrm{a}}$ \\
\hline $\begin{array}{l}\text { Cyclopogon congestus (Vell.) } \\
\text { Hoehne }\end{array}$ & $\mathrm{R} /(\mathrm{T} / \mathrm{E})$ & $\begin{array}{l}\text { FMES/ } \\
\text { FMESA/LR }\end{array}$ & $R^{\text {Ago-Set }}$ & MC & $97 / 72^{\mathrm{a}}, 905^{\mathrm{a}}, 1080^{\mathrm{a}}$ \\
\hline Cyclopogon elatus (Sw.) Schltr. & $\mathrm{T} / \mathrm{R} / \mathrm{E}$ & FMESA & Ago-Set & $\mathrm{C}$ & $\begin{array}{l}97 / 74^{\mathrm{a}}, 705^{\mathrm{a}}, 903^{\mathrm{a}}, \\
1094^{\mathrm{a}}, 1155^{\mathrm{a}}, 1076^{\mathrm{a}}, \\
1080^{\mathrm{a}}, 1088^{\mathrm{a}}, 1090^{\mathrm{a}}\end{array}$ \\
\hline Cyclopogon variegatus Barb. Rodr. & $\mathrm{T}$ & $\begin{array}{l}\text { FMES/ } \\
\text { FMESA }\end{array}$ & Ago-Set & PC & $\begin{array}{l}97 / 71^{\mathrm{a}}, 555^{\mathrm{a}}, 906^{\mathrm{a}} \\
1162^{\mathrm{a}}, 1072^{\mathrm{a}}\end{array}$ \\
\hline Dryadella aviceps (Rchb.f.) Luer & $\mathrm{E}$ & FMES & Out-Dez & $\mathrm{RR}$ & $97 / 89^{\mathrm{a}}, 694^{\mathrm{a}}, 736^{\mathrm{a}}$ \\
\hline Encyclia patens Hook. & $\mathrm{E}$ & $\begin{array}{l}\text { FMES/ } \\
\text { FMESA }\end{array}$ & Mai-Ago & MC & $199^{\mathrm{a}}, 849^{\mathrm{a}}, 1071^{\mathrm{a}}$ \\
\hline Epidendrum armeniacum Lindl. & $\mathrm{E}$ & FMES & Jan-Mar & $\mathrm{PC}$ & $408^{\mathrm{a}}$ \\
\hline Epidendrum chlorinum Barb. Rodr. & $\mathrm{E}$ & FMESA & Fev-Mar & PC & $1135^{\mathrm{a}}, 1137^{\mathrm{a}}$ \\
\hline Epidendrum difforme Jacq. & $\mathrm{E}$ & FMESA & Abr-Jun & $\mathrm{RR}$ & $1242^{\mathrm{a}}$ \\
\hline Epidendrum henschenii Barb. Rodr. & $\mathrm{E}$ & $\begin{array}{l}\text { FMES/ } \\
\text { FMESA }\end{array}$ & Fev-Mar & MR & $561^{\mathrm{a}}, 999^{\mathrm{a}}$ \\
\hline
\end{tabular}




\begin{tabular}{|c|c|c|c|c|c|}
\hline Espécies & $\begin{array}{l}\text { Forma } \\
\text { de vida }\end{array}$ & Habitat & Floração & Ocorrência & $\begin{array}{l}\text { Material } \\
\text { testemunho }\end{array}$ \\
\hline Epidendrum latilabre Lindl. & $\mathrm{E}$ & FMESA & Jan-Fev & $\mathrm{PC}$ & $151^{\mathrm{a}}, 993^{\mathrm{a}}$ \\
\hline Epidendrum martianum Lindl. & $\mathrm{R}$ & FMESA & Fev-Mar & $\mathrm{RR}$ & $1130^{\mathrm{a}}, 1132^{\mathrm{a}}$ \\
\hline Epidendrum ochroclorum Barb. Rodr. & dr. E & FMESA & Abr-Jun & $\mathrm{PC}$ & $567^{\mathrm{a}}, 1065^{\mathrm{a}}$ \\
\hline Epidendrum paniculatum Ruiz \& Pav. & av. R & FMES & Set-Out & $\mathrm{C}$ & $97 / 70^{\mathrm{a}}$ \\
\hline Epidendrum proligerum Barb. Rodr. & Ir. E & FMESA & Abr-Mai & $\mathrm{PC}$ & $1148^{\mathrm{a}}, 1149^{\mathrm{a}}$ \\
\hline Epidendrum secundum Jacq. & $\mathrm{R} / \mathrm{T} /(\mathrm{E})$ & FMES & Ano todo & $\mathrm{MC}$ & $97 / 68^{\mathrm{a}}, 190^{\mathrm{a}}, 181^{\mathrm{a}}$ \\
\hline Eulophia alta (L.) Fawc. \& Rendle & $\mathrm{T}$ & LP & Fev-Mar & $\mathrm{PC}$ & $412^{\mathrm{a}}$ \\
\hline $\begin{array}{l}\text { Eurystyles actinosophila (Barb. } \\
\text { Rodr.) Schltr. }\end{array}$ & $\mathrm{E}$ & $\begin{array}{l}\text { FMES/ } \\
\text { FMESA }\end{array}$ & Fev-Abr & $\mathrm{PC}$ & $186^{\mathrm{a}}, 1154^{\mathrm{a}}$ \\
\hline Galeandra beyrichii Rchb.f. & $\mathrm{T}$ & $\begin{array}{l}\text { FMES/ } \\
\text { FMESA }\end{array}$ & Jan-Fev & $\mathrm{RR}$ & $\begin{array}{l}97 / 96^{\mathrm{a}}, 1114^{\mathrm{a}}, 1117^{\mathrm{a}} \\
1133^{\mathrm{a}}\end{array}$ \\
\hline $\begin{array}{l}\text { Gomesa crispa (Lindl.) } \\
\text { Klotzsch ex Rchb.f. }\end{array}$ & $\mathrm{R} / \mathrm{E} /(\mathrm{T})$ & $\begin{array}{l}\text { FMES/ } \\
\text { FMESA }\end{array}$ & Abr-Jun & $\mathrm{MC}$ & $198^{\mathrm{a}}, 1066^{\mathrm{a}}$ \\
\hline Gomesa recurva R.Br. & $\mathrm{E}$ & FMES/MG & Nov-Jan & $\mathrm{C}$ & $369^{\mathrm{a}}, 1099^{\mathrm{a}}, 1109^{\mathrm{a}}$ \\
\hline Govenia utriculata (Sw.) Lindl. & $\mathrm{T}$ & FMES/LP & Nov-Jan & $\mathrm{C}$ & $97 / 97^{\mathrm{a}}, 1112^{\mathrm{a}}$ \\
\hline Grobya amherstiae Lindl. & $\mathrm{E}$ & FMESA & Fev-Mar & $\mathrm{MC}$ & $566^{\mathrm{a}}, 47^{\mathrm{b}}$ \\
\hline Habenaria araneiflora Barb. Rodr. & T & $\mathrm{LP}$ & Dez-Jan & MR & $565^{\mathrm{a}}$ \\
\hline Habenaria glaucophylla Barb. Rodr & dr. T & FMES & Nov-Mar & $\mathrm{PC}$ & $138^{\mathrm{a}}, 410^{\mathrm{a}}$ \\
\hline Habenaria johannensis Barb. Rodr. & r. $\mathrm{T}$ & FMES/LP & Jan-Mar & $\mathrm{RR}$ & $990^{\mathrm{a}}$ \\
\hline Habenaria josephensis Barb. Rodr. & : $\mathrm{T}$ & FMES/LP & Fev-Abr & $\mathrm{C}$ & $149^{\mathrm{a}}, 847^{\mathrm{a}}, 994^{\mathrm{a}}, 1134^{\mathrm{a}}$ \\
\hline Habenaria parviflora Lindl. & $\mathrm{T}$ & LP & Fev-Abr & $\mathrm{MC}$ & $564^{\mathrm{a}}, 179^{\mathrm{a}}, 671^{\mathrm{c}}$ \\
\hline $\begin{array}{l}\text { Habenaria paulistana Batista } \\
\text { \& Bianchetti }\end{array}$ & $\mathrm{T}$ & FMES/LP & Dez-Jan & MR & $559^{\mathrm{a}}, 726^{\mathrm{a}}$ \\
\hline $\begin{array}{l}\text { Habenaria pleiophylla Hoehne } \\
\text { \& Schltr. }\end{array}$ & $\mathrm{T}$ & LP & Fev-Mai & $\mathrm{PC}$ & $704^{\mathrm{a}}, 180^{\mathrm{a}}, 675^{\mathrm{c}}$ \\
\hline Habenaria riedelii Cogn. & $\mathrm{T}$ & LP & Fev-Abr & MR & $143^{\mathrm{a}}, 706^{\mathrm{a}}, 995^{\mathrm{a}}$ \\
\hline Habenaria sp. & $\mathrm{T}$ & FMESA & Jan & MR & $731^{\mathrm{a}}$ \\
\hline Hapalorchis lineatus (Lindl.) Schltr. & r. $T /(E / R)$ & $\begin{array}{l}\text { FMES/ } \\
\text { FMESA/LP }\end{array}$ & Ago & MC & $\begin{array}{l}843^{\mathrm{a}}, 1158^{\mathrm{a}}, 1082^{\mathrm{a}} \\
1089^{\mathrm{a}}\end{array}$ \\
\hline $\begin{array}{l}\text { Hapalorchis micranthus } \\
\text { (Barb. Rodr.) Hoehne }\end{array}$ & $\mathrm{T}$ & $\begin{array}{l}\text { LP/ } \\
\text { FMESA }\end{array}$ & Ago & $\mathrm{PC}$ & $842^{\mathrm{a}}, 1159^{\mathrm{a}}$ \\
\hline $\begin{array}{l}\text { Heterotaxis brasiliensis } \\
\text { (Brieger \& Illg) F.Barros }\end{array}$ & $\mathrm{R}$ & FMES & Abr-Mai & $\mathrm{PC}$ & $841^{\mathrm{a}}, 1059^{\mathrm{a}}$ \\
\hline Ionopsis utricularioides (Sw.) Lindl. & 1. $\mathrm{E}$ & FMESA & Ago-Set & MR & $1067^{\mathrm{a}}$ \\
\hline $\begin{array}{l}\text { Isabelia violacea (Lindl.) } \\
\text { Van den Berg \& M.W. Chase }\end{array}$ & $\mathrm{R} /(\mathrm{E})$ & $\begin{array}{l}\text { LR/ } \\
\text { FMESA }\end{array}$ & Jul-Ago & $\mathrm{RR}$ & $836^{\mathrm{a}}$ \\
\hline Isabelia virginalis Barb. Rodr. & $\mathrm{E}$ & FMES & Mai-Jun & $\mathrm{RR}$ & $924^{\mathrm{a}}$ \\
\hline Isochilus linearis (Jacq.) R.Br. & $\mathrm{E} / \mathrm{R}$ & FMES/MG & Out-Mar & $\mathrm{PC}$ & $97 / 98^{\mathrm{a}}, 1139^{\mathrm{a}}$ \\
\hline Liparis nervosa (Thunb.) Lindl. & $\mathrm{T}$ & FMES & Dez-Jan & $\mathrm{PC}$ & $97 / 100^{\mathrm{a}}, 1111^{\mathrm{a}}$ \\
\hline Lockhartia lunifera (Lindl.) Rchb.f. & - $E$ & FMES & Dez-Fev & $\mathrm{PC}$ & $696^{\mathrm{a}}, 1113^{\mathrm{a}}, 1115^{\mathrm{a}}$ \\
\hline Malaxis excavata (Lindl.) Kuntze & $\mathrm{T} / \mathrm{R}$ & FMES & Jan-Mar & MR & $1086^{\mathrm{a}}$ \\
\hline Maxillaria notylioglossa Rchb.f. & $\mathrm{E}$ & FMES & Set-Nov & $\mathrm{PC}$ & $1240^{\mathrm{a}}$ \\
\hline
\end{tabular}




\begin{tabular}{|c|c|c|c|c|c|}
\hline Espécies & $\begin{array}{l}\text { Forma } \\
\text { de vida }\end{array}$ & Habitat & Floração & Ocorrência & $\begin{array}{l}\text { Material } \\
\text { testemunho }\end{array}$ \\
\hline Maxillaria leucaimata Barb. Rodr. & r. $\mathrm{E}$ & FMES & Out-Nov & $\mathrm{RR}$ & $770^{\mathrm{a}}, 929^{\mathrm{a}}$ \\
\hline Mesadenella cuspidata (Lindl.) Garay & ay $\mathrm{T}$ & FMES & Fev-Mar & $\mathrm{MC}$ & $146^{\mathrm{a}}, 1126^{\mathrm{a}}$ \\
\hline Miltonia regnellii Rchb.f. & $\mathrm{E}$ & MG/FMES & Jan-Mar & $\mathrm{RR}$ & $844^{\mathrm{a}}$ \\
\hline Notylia nemorosa Barb. Rodr. & $\mathrm{E}$ & FMES & Jul-Ago & $\mathrm{RR}$ & $1241^{\mathrm{a}}$ \\
\hline Octomeria crassifolia Lindl. & E & FMESA & Abr & PC & $1062^{\mathrm{a}}$ \\
\hline Octomeria diaphana Lindl. & $\mathrm{E}$ & FMES & Nov-Mai & PC & $\begin{array}{l}97 / 87^{\mathrm{a}}, 549^{\mathrm{a}}, 1102^{\mathrm{a}} \\
1063^{\mathrm{a}}, 1125^{\mathrm{a}}\end{array}$ \\
\hline Octomeria fasciculata Barb. Rodr. & $\mathrm{E}$ & FMESA & Set-Mai & PC & $316^{\mathrm{a}}, 317^{\mathrm{a}}, 1064^{\mathrm{a}}$ \\
\hline Oeceoclades maculata (Lindl.) Lindl. & dl. $\mathrm{T}$ & FMES/LP & Mar-Abr & $\mathrm{C}$ & $137^{\mathrm{a}}, 140^{\mathrm{a}}, 996^{\mathrm{a}}$ \\
\hline Oncidium crispum Lodd. ex Lindl. & $\mathrm{E}$ & FMES & Ago-Set & $\mathrm{RR}$ & $1243^{\mathrm{a}}$ \\
\hline Oncidium flexиosum Lodd. & E & FMESA & Nov-Dez & PC & $1105^{\mathrm{a}}$ \\
\hline Oncidium harrisonianum Lindl. & $\mathrm{E}$ & FMES & Jan-Fev & $\mathrm{RR}$ & $701^{\mathrm{a}}$ \\
\hline Oncidium hians Lindl. & $\mathrm{E}$ & FMES & Fev-Mar & PC & $560^{\mathrm{a}}, 670^{\mathrm{a}}$ \\
\hline Oncidium hookeri Rolfe & $\mathrm{E}$ & FMESA & Fev-Mar & $\mathrm{RR}$ & $568^{\mathrm{a}}, 988^{\mathrm{a}}$ \\
\hline Oncidium longipes Lindl. & $\mathrm{E}$ & FMES & Out-Nov & PC & $725^{\mathrm{a}}, 1103^{\mathrm{a}}$ \\
\hline Oncidium montanum Barb. Rodr. & $\mathrm{R} /(\mathrm{T})$ & FMESA/LR & R Jan-Mar & $\mathrm{RR}$ & $989^{a}$ \\
\hline Oncidium praetextum Rchb.f. & $\mathrm{E}$ & FMESA & Abr-Mai & $\mathrm{C}$ & $850^{\mathrm{a}}, 1150^{\mathrm{a}}$ \\
\hline Oncidium varicosum Lindl. \& Paxton & on $\mathrm{E}$ & $\begin{array}{l}\text { FMES/ } \\
\text { FMESA }\end{array}$ & Abr-Jun & $\mathrm{C}$ & $925^{\mathrm{a}}$ \\
\hline $\begin{array}{l}\text { Pelexia oestrifera (Rchb.f. } \\
\text { \& Warm.) Schltr. }\end{array}$ & $\mathrm{T} / \mathrm{R}$ & FMES & Jul-Ago & $\mathrm{PC}$ & $904^{\mathrm{a}}$ \\
\hline Pleurothallis schenkii (Cogn.) Luer & er E & FMES & Fev-Mar & $\mathrm{C}$ & $413^{\mathrm{a}}, 703^{\mathrm{a}}, 1140^{\mathrm{a}}$ \\
\hline Polystachya caespitosa Barb. Rodr. & r. E & FMES & Jan-Mar & MR & $716^{\mathrm{a}}, 991^{\mathrm{a}}$ \\
\hline Polystachya estrellensis Rchb.f. & $\mathrm{E} /(\mathrm{R})$ & FMES & Jan-Fev & $\mathrm{MC}$ & $702^{\mathrm{a}}, 992^{\mathrm{a}}, 1131^{\mathrm{a}}$ \\
\hline Prescottia colorans Lindl. & $\mathrm{T}$ & FMESA & Set-Out & $\mathrm{RR}$ & $835^{\mathrm{a}}, 1161^{\mathrm{a}}$ \\
\hline Prescottia montana Barb. Rodr. & $\mathrm{T}$ & $\mathrm{LR} / \mathrm{LP}$ & Ago-Set & PC & $1156^{\mathrm{a}}$ \\
\hline Prescottia oligantha Lindl. & $\mathrm{T}$ & FMESA & Ago & PC & $1157^{\mathrm{a}}, 1087^{\mathrm{a}}$ \\
\hline Prescottia stachyodes (Sw.) Lindl. & $\mathrm{T}$ & $\begin{array}{l}\text { FMES/ } \\
\text { FMESA }\end{array}$ & Ago-Out & $\mathrm{C}$ & $\begin{array}{l}835^{\mathrm{a}}, 1161^{\mathrm{a}}, 97 / 69^{\mathrm{a}}, \\
1084^{\mathrm{a}}\end{array}$ \\
\hline Promenaea rollissonii (Lindl.) Lindl. & 11. E & FMESA & $\mathrm{Fev}$ & MR & $1244^{\mathrm{a}}$ \\
\hline $\begin{array}{l}\text { Prosthechea bulbosa (Vell.) } \\
\text { W.E.Higgins }\end{array}$ & $\mathrm{E} /(\mathrm{R})$ & $\begin{array}{l}\text { FMESA/ } \\
\text { FMES }\end{array}$ & Fev-Mar & $\mathrm{PC}$ & $732^{\mathrm{a}}, 1136^{\mathrm{a}}$ \\
\hline $\begin{array}{l}\text { Prosthechea calamaria (Lindl.) } \\
\text { W.E. Higgins }\end{array}$ & $\mathrm{E}$ & FMES & Abr-Mai & PC & $562^{\mathrm{a}}$ \\
\hline Psilochilus modestus Barb. Rodr. & $\mathrm{T}$ & FMES & Dez-Mar & PC & $132^{\mathrm{a}}, 838^{\mathrm{a}}$ \\
\hline $\begin{array}{l}\text { Pteroglossa glazioviana (Cogn.) } \\
\text { Garay }\end{array}$ & $\mathrm{T}$ & FMES & Nov-Dez & $\mathrm{RR}$ & $557^{\mathrm{a}}$ \\
\hline $\begin{array}{l}\text { Rodriguezia decora (Lem.) } \\
\text { Rchb.f. }\end{array}$ & $\mathrm{E} / \mathrm{R}$ & $\begin{array}{l}\text { LR/FMES/ } \\
\text { FMESA }\end{array}$ & / Mar-Abr & $\mathrm{C}$ & $222^{\mathrm{a}}, 182^{\mathrm{a}}$ \\
\hline $\begin{array}{l}\text { Rodrigueziella gomezoides } \\
\text { (Barb. Rodr.) Berman }\end{array}$ & $\mathrm{E}$ & FMESA & Abr-Mai & MR & $771^{\mathrm{a}}$ \\
\hline $\begin{array}{l}\text { Rodrigueziella handroi } \\
\text { (Hoehne) Pabst }\end{array}$ & $\mathrm{E}$ & FMESA & Mar-Abr & $\mathrm{RR}$ & $1146^{\mathrm{a}}$ \\
\hline
\end{tabular}




\begin{tabular}{|c|c|c|c|c|c|}
\hline Espécies & $\begin{array}{l}\text { orma } \\
\text { le vida }\end{array}$ & Habitat & Floração & Ocorrência & $\begin{array}{l}\text { Material } \\
\text { testemunho }\end{array}$ \\
\hline Sarcoglottis fasciculata (Vell.) Schltr. & tr. $\mathrm{T}$ & FMES/LP & Ago-Set & $\mathrm{PC}$ & $1079^{\mathrm{a}}, 1091^{\mathrm{a}}$ \\
\hline Sauroglossum nitidum (Vell.) Schltr. & r. $\mathrm{T}$ & $\begin{array}{l}\text { FMES/ } \\
\text { FMESA }\end{array}$ & Jul-Set & MC & $97 / 73^{\mathrm{a}}, 1070^{\mathrm{a}}, 1077^{\mathrm{a}}$ \\
\hline Specklinia ephemera (Lindl.) Luer & E & FMES & Mar-Mai & $\mathrm{RR}$ & $141^{\mathrm{a}}$ \\
\hline $\begin{array}{l}\text { Specklinia grobyi (Bateman } \\
\text { ex Lindl.) F.Barros }\end{array}$ & $\mathrm{E} / \mathrm{R}$ & FMES & Fev-Mai & MC & $404^{\mathrm{a}}, 1061^{\mathrm{a}}, 1116^{\mathrm{a}}, 1141^{\mathrm{a}}$ \\
\hline $\begin{array}{l}\text { Specklinia hypnicola (Lindl.) } \\
\text { F.Barros }\end{array}$ & $\mathrm{E}$ & FMES & Mar-Ago & MC & $1143^{\mathrm{a}}, 1152^{\mathrm{a}}$ \\
\hline $\begin{array}{l}\text { Specklinia uniflora (Lindl.) } \\
\text { Pridgeon \& M.W. Chase }\end{array}$ & $\mathrm{E}$ & FMES & Fev-Mar & $\mathrm{C}$ & $142^{\mathrm{a}}, 1142^{\mathrm{a}}$ \\
\hline Stanhopea lietzei (Regel) Schltr. & $\mathrm{R}$ & FMES & Out-Dez & MR & $97 / 88^{\mathrm{a}}, 1108^{\mathrm{a}}$ \\
\hline $\begin{array}{l}\text { Stenorrhynchos lanceolatum } \\
\text { (Aubl.) Rich. ex Spreng. }\end{array}$ & $\mathrm{R}$ & $\mathrm{LR} / \mathrm{LP}$ & Set-Nov & $\mathrm{PC}$ & $550^{\mathrm{a}}, 1092^{\mathrm{a}}$ \\
\hline $\begin{array}{l}\text { Trichocentrum pumilum (Lindl.) } \\
\text { M.W.Chase \& N.H.Williams }\end{array}$ & $\mathrm{E}$ & FMES & Dez-Jan & $\mathrm{C}$ & $97 / 99^{\mathrm{a}}$ \\
\hline Vanilla bahiana Hoehne & $\mathrm{HE}$ & FMES & Out-Nov & $\mathrm{PC}$ & $97 / 78^{\mathrm{a}}, 727^{\mathrm{a}}$ \\
\hline Vanilla edwallii Hoehne & $\mathrm{HE}$ & FMES & Nov-Jan & $\mathrm{C}$ & $407^{\mathrm{a}}, 695^{\mathrm{a}}, 840^{\mathrm{a}}$ \\
\hline Wullschlaegelia aphylla (Sw.) Rchb.f. & f. $S$ & FMES & Dez-Jan & $\mathrm{C}$ & $130^{\mathrm{a}}, 707^{\mathrm{a}}, 1000^{\mathrm{a}}, 1118^{\mathrm{a}}$ \\
\hline Zygopetalum mackayi Hook. & $\mathrm{R} / \mathrm{T}$ & FMESA/LR & Nov-Mar & $\mathrm{C}$ & $721^{\mathrm{a}}, 1127^{\mathrm{a}}$ \\
\hline Zygostates lunata Lindl. & $\mathrm{E}$ & FMES & Out-Nov & MR & $558^{\mathrm{a}}$ \\
\hline
\end{tabular}

${ }^{\mathrm{a}}$ E.R. Pansarin, ${ }^{\mathrm{b}}$ L. Mickeliunas, ${ }^{\mathrm{c}}$ L.Y.S. Aona

\section{Resultados}

Orchidadeae está representada na Serra do Japi por 125 espécies distribuídas em 61 gêneros pertencentes a três subfamílias: Epidendroideae (93 spp., 74,4\%), Orchidoideae (30 spp., 24\%) e Vanilloideae (2 spp., 1,6\%). Os gêneros mais representativos são: Epidendrum (10 spp.), Habenaria e Oncidium (9 spp. cada), e Cyclopogon (6 spp.) (Tab. 1). Dentre as espécies que ocorrem na Serra do Japi, a grande maioria (79 spp., 63,2\%) é encontrada como epífita, 40 (32\%) são terrícolas, 31 (24,8\%) são rupícolas, duas são hemiepífitas e apenas uma, Wullschlaegelia aphylla, é saprofítica (Fig. 2). Dentre as espécies que ocorrem como epífitas, cerca de $30 \%$ podem ocorrer ocasionalmente como rupícolas ou, mais raramente, terrícolas (Tab. 1). Hapalorchis lineatus, por exemplo, pode ser encontrada como terrícola, rupícola e epífita. O ambiente com maior número de espécies na Serra do Japi é o que apresenta predominância de floresta mesófila estacional semidecídua (88 spp., 70,9\%), seguido por áreas de floresta mesófila estacional semidecídua de altitude (46 spp., 37,1\%). Os lajeados rochosos são áreas com baixa riqueza específica na Serra do Japi, ocorrendo apenas 12 espécies $(9,7 \%)$ nesse tipo de vegetação (Fig. 3). No entanto, os lajeados rochosos podem apresentar espécies que são encontradas principalmente nesse tipo de formação, como Bifrenaria harrisoniae, Rodriguezia decora, Pelexia oestrifera e Oncidium montanum. Várias espécies podem ocorrer em dois ou mais tipos de formação, como Cyclopogon congestus, por exemplo, que pode ser encontrado por toda a Serra do Japi (Tab. 1). A maioria das espécies floresce entre os meses de novembro e abril, principalmente entre fevereiro e março (Fig. 4), que corresponde ao verão, período mais chuvoso na Região Sudeste. Entre os meses de maio e julho (estação seca), um menor número de espécies encontra-se em floração (Fig. 4), entre elas Brasiliorchis picta e Isabelia virginalis (Tab. 1). 


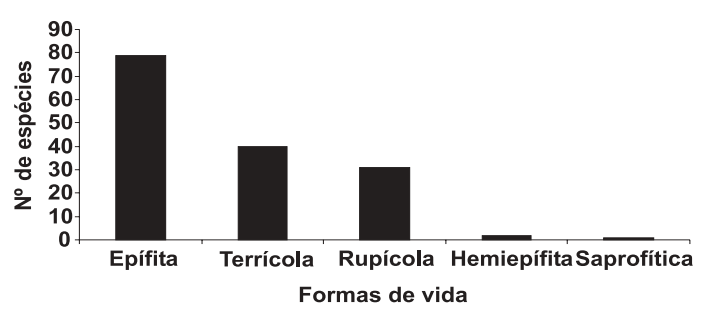

Figura 2 - Número de espécies de Orchidaceae em relação aos diferentes tipos de formas de vida encontrados na Serra do Japi.

\section{Discussão}

Os gêneros mais representativos na Serra do Japi, Epidendrum e Oncidium, são também os mais comuns em áreas de floresta ombrófila do sudeste do Brasil (Barros 1983, 1991; Miller \& Warren 1994). Gêneros como Habenaria, que são ricos em regiões de cerrado do Distrito Federal (Batista \& Bianchetti 2003; Batista et al. 2005), principalmente porque a maioria das espécies ocorre em áreas de campo, geralmente são pouco representativos em regiões com predominância de florestas (e.g. Barros 1983; 1991; Ribeiro 1992; Miller \& Warren 1994). Embora a Serra do Japi tenha predominância de áreas de florestas, a maioria das espécies de Habenaria que ocorre na região é encontrada em locais perturbados (Tab. 1). Alguns gêneros (e.g. Xylobium e Huntleya), no entanto, que são comuns em regiões de mata, e que ocorrem em áreas de florestas mesófilas estacionais semidecíduas de Minas Gerais (Menini Neto et al. 2004a, b) e regiões de floresta ombrófila de São Paulo (Barros 1991), não foram encontrados na região estudada, embora a Serra do Japi seja considerada uma região ecotonal e tida como apresentando um número elevado de espécies de ambas as formações (Leitão Filho 1992).

Orchidaceae é considerada uma das famílias mais representativas em estudos de levantamento de epífitos vasculares em regiões neotropicais (revisão em Kersten \& Silva 2001). O maior número de espécies ocorrendo como epífita é comum em estudos de florística envolvendo a família Orchidaceae em formações florestais inseridas no domínio atlântico, como nas florestas ombrófilas do Rio de Janeiro (Miller \& Warren 1994) e São Paulo (Barros 1991; Ribeiro 1992), nas florestas mesófilas estacionais semidecíduas de Minas Gerais (Menini Neto et al. 2004a, b), e restingas do Espírito Santo (Fraga \& Peixoto 2004), assim como na região amazônica (Silva et al. 1995), contrastando com a maioria de espécies terrícolas que podem ser encontradas em regiões com predominância de cerrado como, por exemplo, no Distrito Federal (e.g. Batista \& Bianchetti 2003; Batista et al. 2005).

De acordo com Waechter (1986), as vantagens proporcionadas pelo epifitismo são as melhores condições de luminosidade e substrato relativamente isento de competição. O epifitismo é responsável por parte significativa da diversidade que faz das florestas tropicais úmidas um dos mais complexos ecossistemas da Biosfera, constituindo até $50 \%$ do total de espécies vasculares. A capacidade destas florestas em sustentar grande número de animais

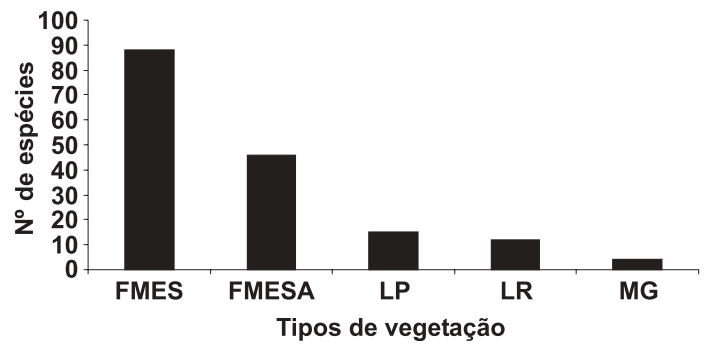

Figura 3 - Número de espécies de Orchidaceae em relação aos tipos de vegetação encontrados na Serra do Japi. FMES = floresta mesófila estacional semidecídua, FMESA = floresta mesófila estacional semidecídua de altitude, $\mathrm{LR}=$ lajeado rochoso, $\mathrm{LP}=$ local perturbado, $\mathrm{MG}=$ mata de galeria.

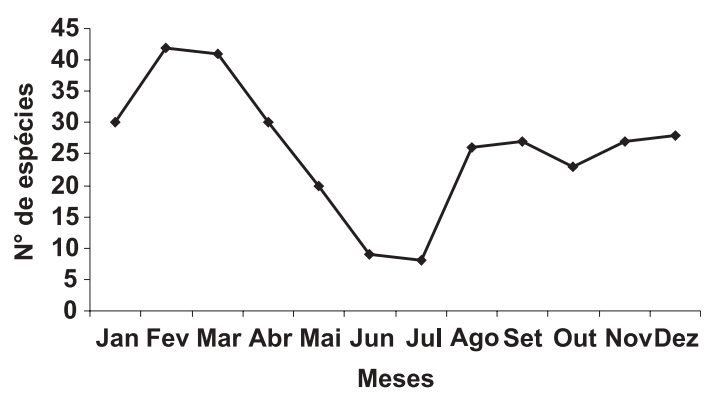

Figura 4 - Número de espécies de Orchidaceae em floração em cada mês do ano na Serra do Japi. 
pode ser atribuída ao substrato e sustento providos pelas epífitas, e por sua respectiva capacidade de retenção de nutrientes da chuva, neblina e partículas em suspensão (Nadkarni 1986).

Entre as espécies que ocorrem na Serra do Japi, cerca de $5 \%$ são encontradas no cerrado de São Paulo (Cardoso \& Israel 2005), 10\% em regiões de restinga do Espírito Santo (Fraga \& Peixoto 2004), mais de $15 \%$ em florestas mesófilas estacionais semidecíduas de Minas Gerais (e.g. Menini Neto et al. 2004a, b), quase $20 \%$ ocorrem em regiões de cerrado do Distrito Federal (Batista \& Bianchetti 2003; Batista et al. 2005) e 23\% na Chapada Diamantina (Toscanode-Brito \& Cribb 2005). Em áreas de floresta ombrófila da Região Sudeste do Brasil, $43 \%$ das espécies encontradas na Serra também ocorrem na região de Macaé de Cima (Nova Friburgo - RJ) (Miller \& Warren 1994) e 26,4\% podem ser encontradas na Ilha do Cardoso (Cananéia - SP) (Barros 1983, 1991). As duas espécies que não puderam ser identificadas, Habenaria sp. e Acianthera sp., aparentemente são novas para a ciência.

Algumas espécies que ocorrem na Serra do Japi, como Isochilus linearis, Hapalorchis lineatus, Epidendrum difforme e Ionopsis utricularioides apresentam ampla distribuição, podendo ser encontradas até a América Central (Ackerman 1995). As espécies Eulophia alta, Liparis nervosa, Oeceoclades maculata e Polystachya estrellensis, apresentam distribuição transcontinental, podendo ser encontradas nas Américas e Ásia e/ou África. Das espécies que ocorrem na Serra, $36 \%$ são distribuídas pela América do Sul, 20\% são exclusivas do Brasil, 4\% são endêmicas da Região Sudeste e apenas uma, Habenaria paulistana, ocorre exclusivamente no estado de São Paulo. Ionopsis utricularioides, que é uma espécie encontrada muito raramente na Serra do Japi, é muito comum em áreas de floresta mesófila estacional semidecídua e matas de galeria em regiões adjacentes às áreas de cerrado do interior do estado como, por exemplo, nos municípios de São Carlos, Itirapina, Jaboticabal e Bauru (E.R. Pansarin observações pessoais).
Algumas espécies que ocorrem na Serra do Japi são mais comumente encontradas em áreas de floresta ombrófila, como, por exemplo, Zygostates lunata, que é típica desse tipo de formação (Ribeiro 1992; E. R. Pansarin observações pessoais). Zygostates lunata é uma espécie muito rara na Serra do Japi, sendo encontrada apenas em vales muito úmidos e sombreados. Outra espécie que é muito comum em áreas de floresta ombrófila e restinga é Gongora bufonia (Barros 1991; Ribeiro 1992). Embora indivíduos dessa espécie não tenham sido encontrados na região de estudo, de acordo com Pansarin et al. (2006), através da administração de iscas odores, foram capturados machos de Eufriesea violacea Blanchard 1840 (Apidae: Euglossini) carregando polinários dessa espécie. De acordo com F. Pinheiro (com. pess.), embora Gongora bufonia seja comum em áreas de floresta ombrófila, essa espécie pode ser encontrada até Itirapina, um município com predominância de áreas de cerrado no interior do estado de São Paulo.

\section{Conservação da Serra do Japi}

A existência de espécies que ocorrem em áreas de cerrado e em florestas ombrófilas, pode ser explicada pela posição geográfica da Serra do Japi, que está situada entre as florestas ombrófilas e as florestas mesófilas estacionais semideciduais do planalto paulista, o que caracteriza uma região de transição, permitindo a ocorrência de um número elevado de espécies de ambas as formações (Leitão Filho 1992). A Mata Atlântica é considerada o bioma brasileiro mais rico em espécies de Orchidaceae (Pabst \& Dungs 1975; 1977), fato que pode estar relacionado, principalmente, com a diversidade de ambientes, ecossistemas e precipitação que estão associados a esse bioma (Mantovani 1990; 1998; Ivanauskas et al. 2000; Oliveira Filho \& Fontes 2000; Scudeller et al. 2001; Scarano 2002).

Algumas espécies como Cattleya loddigesii, Stanhopea lietzei e Cirrhaea dependens, por serem muito ornamentais, foram muito coletadas por orquidófilos da região. No entanto, na Serra da Japi ainda 
podem ser encontradas grandes populações das duas primeiras espécies. Cirrhaea dependens não forma populações, sendo encontrados apenas indivíduos isolados em vales sombreados e nas bordas de paredões rochosos (Pansarin et al. 2006).

Além da coleta indiscriminada de orquídeas ornamentais por orquídófilos, o estabelecimento de moradias no interior da Serra e substituição de áreas nativas por bosques de Pinus e Eucalyptus, compromete não somente as espécies de orquídeas que ocorrem na região, mas também o restante da flora e, conseqüentemente, a fauna da Serra do Japi. Esse processo é semelhante ao que vem ocorrendo com outras áreas dentro do bioma da Mata Atlântica, hoje apenas com 58\% de sua formação original (Dean 1995; Morellato \& Haddad 2000). Assim como ocorre com a Serra do Japi, que se encontra rodeada por grandes centros urbanos e sofre com intervenções do homem sob diversos aspectos, os últimos remanescentes de Mata Atlântica do estado de São Paulo também estão sujeitos a intervenções, como especulação imobiliária, incêndios, substituição da mata nativa para estabelecimento de culturas e criação de gado (Dean 1995; Morellato \& Haddad 2000). De acordo com Leitão Filho (1992), somente através do conhecimento da composição florística, estrutura fitossociológica e dinâmica da fitocenose serão possíveis estabelecer modelos seguros para a recuperação de extensas áreas do sudeste do Brasil e resgatar a grande maioria das espécies atualmente ameaçadas de extinção. A preservação e o estudo integrado da Serra do Japi é uma urgente necessidade científica, com reflexos sociais, econômicos e preservacionistas.

\section{Agradecimentos}

Os autores agradecem a Fábio Pinheiro, João A.N. Batista e Wellington Forster pelo auxílio na identificação das espécies; Cássio van den Berg pelas valiosas sugestões; a Base Ecológica da Serra do Japi e Guarda Municipal de Jundiaí pela autorização concedida para realização dos trabalhos de campo.

\section{REFERÊNCIAS BIBLIOGRÁFICAS}

Ackerman, J. D. 1995. An orchid flora of Puerto Rico and the Virgin Islands. New York Botanical Garden, New York, 204p.

Atwood, J. T. 1986. The size of the Orchidaceae and the systematic distribution of epiphytic orchids. Selbyana 9(1): 171-186.

Barros, F. 1983. Flora Fanerogâmica da Reserva do Parque Estadual das Fontes do Ipiranga (São Paulo, Brasil). 198 Orchidaceae. Hoehnea 10: 74-124. 1991. Orchidaceae. In: Melo, M. M. R. F.; Barros, F.; Wanderley, M. G. L.; Kirizawa, M.; Jung-Mendaçolli, S. L. \& Chiea, S. A. C. (eds.). Flora fanerogâmica da Ilha do Cardoso: Caracterização geral da vegetação e listagem das espécies ocorrentes. Instituto de Botânica, São Paulo, 1: 142-152.

1996. Notas taxonômicas para espécies brasileiras dos gêneros Epidendrum, Platystele, Pleurothallis e Scaphyglottis (Orchidaceae). Acta Botanica Brasilica 10(1): 139-151.

Batista, J. A. N. \& Bianchetti, L. B. 2003. Lista atualizada das Orchidaceae doDistrito Federal. Acta Botanica Brasilica 17(2): 183-201.

; Bianchetti, L. B. \& Pellizzaro, K. F. 2005. Orchidaceae da Reserva Ecológica do Guará, DF, Brasil. Acta Botanica Brasilica 19(2): 221-232.

Cardoso, J. C. \& Israel, M. 2005. Levantamento de espécies da família Orchidaceae em Águas de Sta. Bárbara (SP) e seu cultivo. Horticultura Brasileira 23(2): 169-173.

Chase, M. W.; Cameron, K. M.; Barrett, R. L. \& Freudenstein, J. V. 2003. DNA data and Orchidaceae systematics: a new phylogenetic classification. In: Dixon, K. W.; Kell, S. P.; Barrett, R. L. \& Cribb, P. J. (eds.). Orchid Conservation. Natural History Publications, Kota Kinabalu, Sabah. Pp. 69-89.

Christenson, E. A. 1988. Nomenclatural changes in neotropical Orchidaceae. Lindleyana 3(4): 221-223.

.1996. Notes on neotropical Orchidaceae II. Lindleyana 11(1): 12-26. 
Cogniaux, A. 1893-1896. Orchidaceae. In: Martius, C. F. P.; Eichler, A. G. \& Urban, I. (eds.). Flora brasiliensis. Munique, F. Fleischer 3(4): 1-672.

1898-1902. Orchidaceae. In: Martius, C. F. P.; Eichler, A. G. \& Urban, I. (eds.). Flora brasiliensis. Munique, R. Oldenbourg 3(5): 1-664.

1904-1906. Orchidaceae. In: Martius, C. F. P.; Eichler, A. G. \& Urban, I. (eds.). Flora brasiliensis. Munique, R. Oldenbourg 3(6): 1-604.

Dean, W. 1995. A ferro e fogo: a história e a devastação da Mata Atlântica brasileira. Companhia das Letras, São Paulo, 484p.

Dressler, R. L. 1993. Phylogeny and classification of the orchid family. Dioscorides Press, Portland, 314p.

Fraga, C. N. \& Peixoto, A. L. 2004. Florística e ecologia das Orchidaceae da restinga do estado do Espírito Santo. Rodriguésia 55(84): 5-20.

Garay, L. A. 1977. Systematics of the Physurinae (Orchidaceae) in the new world. Bradea 2(28): 191-204.

1980. A generic revision of the Spiranthinae. Botanical Museum Leaflets Harvard University 28(4): 277-425.

Hágsater, E. 1993. Epidendrum anceps or Epidendrum secundum? Orquídea 13(1-2): 153-158.

Hoehne, F. C. 1940. Orchidaceas. In: Hoehne, F. C. (ed.). Flora Brasílica. Secretaria da Agricultura, Indústria e Comércio de São Paulo, São Paulo, 12(1): 1-254.

1942. Orchidaceas. In: Hoehne, F. C. (ed.). Flora Brasílica. Secretaria da Agricultura, Indústria e Comércio, São Paulo, 12(6): 1-218.

1945. Orchidaceas. In: Hoehne, F. C. (ed.). Flora Brasílica. Secretaria da Agricultura, Indústria e Comércio, São Paulo, 12(2): 1-389.

1949. Iconografia de orquidáceas do Brasil. S. A. Indústrias "Graphicars-f. Lanzara”, São Paulo, 601p.
1953. Orchidaceas. In: Hoehne, F. C. (ed.). Flora Brasílica. Secretaria da Agricultura, Indústria e Comércio de São Paulo, São Paulo, 12(7): 1-397.

Ivanauskas, N. M.; Monteiro, R. \& Rodrigues, R. R. 2000. Similaridade florística entre áreas de floresta Atlântica no estado de São Paulo. Brazilian Journal of Ecology 1(4): 71-81.

Kersten, R. A. \& Silva, S. M. 2001. Composição florística e estrutura do componente epifítico vascular em floresta da planície litorânea na Ilha do Mel, Paraná, Brasil. Revista Brasileira de Botânica 24(2): 213-226.

Leitão Filho, H. F. 1992. A flora arbórea da Serra do Japi. In: Morellato, L. P. C. (org.). História natural da Serra do Japi: Ecologia e preservação de uma área florestal no sudeste do Brasil. Editora da Unicamp/Fapesp, Campinas, Pp. 40-62.

Mantovani, W. 1990. A dinâmica das florestas de encosta Atlântica. In: Anais do II Simpósio de Ecossistemas da Costa Sul e Sudeste Brasileira, São Paulo. Pp. 304-313. 1998. Dinâmica da Floresta Pluvial Atlântica. In: Anais do IV Simpósio de Ecossistemas Brasileiros. ACIESP, Águas de Lindóia. Pp. 1-20.

Menini Neto, L.; Assis, L. C. S. \& Forzza, R. C. 2004a. A família Orchidaceae em um fragmento de floresta estacional semidecidual, no município de Barroso, Minas Gerais, Brasil. Lundiana 5(1): 9-27. ; Almeida, V.R. \& Forzza, R. C. 2004b. A família Orchidaceae na Reserva Biológica da Represa do Gama - Descoberto, Minas Gerais, Brasil. Rodriguésia 55(84): 137-156.

Miller, D. \& Warren, R. 1994. Orchids of the high mountain Atlantic rain forest in southeastern Brazil. Riode Janeiro, Salamandra, 182p.

Morellato, L. P. C. 1992. História natural da Serra do Japi: Ecologia e preservação de uma área florestal no sudeste do Brasil. Editora da Unicamp/Fapesp, Campinas, 321p.

$\&$ Haddad, C. F. B. 2000. Introduction: The Brazilian Atlantic Forest. Biotropica 32(4b): 786-792. 
Nadkarni, N. M. 1986. An ecological overview and checklist of vascular epiphytes in the Monteverde cloud forest reserve, Costa Rica. Brenesia 24(1): 55-632.

Oliveira Filho, A. T. \& Machado, J. M. N. 1993. Composição florística de uma floresta semidecídua montana na Serra de São José, Tiradentes, Minas Gerais. Acta Botanica Brasilica 7(2): 71-88. ; Scolforo, J. R. S. \& Melo, J. M. 1994. Composição florística e estrutura comunitária de um remanescente de floresta semidecídua montana em Lavras, MG. Revista Brasileira de Botânica 17(2): 167-182.

\& Fontes, M. A. L. 2000. Patterns of floristic differentiation among Atlantic Forests in Southeastern Brazil, and the influence of climate. Biotropica 32(4b): 793-810.

Pabst, G. F. J. 1950. Notas sôbre "Polystachya estrellensis, Rchb. f.".'. Orquidea (Rio de Janeiro) 12(1): 167-169.

1977. Orchidaceae Brasilienses. Vol.

2. Kurt Schmersow, Hildesheim, 418p.

\& Dungs, F. 1975. Orchidaceae

Brasilienses. Vol. 1. Kurt Schmersow, Hildesheim, 408p.

Pansarin, E. R.; Bittrich, V. \& Amaral, M. C. E. 2006. At daybreak - reproductive biology and isolating mechanisms of Cirrhaea dependens (Orchidaceae). Plant Biology 8(4): 494-502.

Pinto, H. S. 1992. O clima da Serra do Japi. In: Morellato, L. P. C. (org.). História natural da Serra do Japi: Ecologia e preservação de uma área florestal no sudeste do Brasil. Editora da Unicamp/Fapesp, Campinas. Pp. 30-38.
Ribeiro, J. E. 1992. Florística e padrões de distribuição da família Orchidaceae na planície litorânea do núcleo de desenvolvimento Picinguaba, município de Ubatuba, Parque Estadual da Serra do Mar, SP. Dissertação de Mestrado. Universidade Estadual Paulista, Rio Claro, 304p.

Scarano, F. R. 2002. Structure, function and floristic relationships of plant communities in stressful habitats marginal to the Brazilian Atlantic rainforest. Annals of Botany 90(4): 517-524.

Scudeller, V. V.; Martins, F. R. \& Shepherd, G. J. 2001. Distribution and abundance of arboreal species in the Atlantic ombrophilous dense forest in Southeastern Brazil. Plant Ecology 152(2): 185-199.

Silva, M. F. F.; Silva, J. B. F.; Rocha, A. E. S.; Oliveira, F. P. M.; Gonçalves, L. S. B.; Silva, M. F. \& Queiroz, O. H. A. 1995. Inventário da família Orchidaceae na Amazônia brasileira. Parte I. Acta Botanica Brasilica 9(1): 163-175.

Sprunger, S.; Cribb, P. \& Toscano de Brito, A. L. V. 1996. João Barbosa Rodrigues - Iconographie des orchidées du Brésil. Vol. 1. The illustrations. Friedrich Reinhardt, Basle, 324p.

Toscano-de-Brito, A. L. V. \& Cribb, P. 2005. Orquídeas da Chapada Diamantina. Nova Fronteira, Rio de Janeiro, 400p.

van den Berg, C. \& Chase, M. W. 2001. Nomenclatural notes on Laeliinae-II. Additional combinations and notes. Lindleyana 16(2): 109-112.

Waechter, J. L. 1986. Epífitos vasculares da mata paludosa do Faxinal, Torres, Rio Grande do Sul, Brasil. Iheringia, Série Botânica 34(1): 39-49. 